\title{
Abnormalities of T cell signaling in systemic lupus erythematosus
}

\author{
Vaishali R Moulton* and George C Tsokos
}

\begin{abstract}
Systemic lupus erythematosus (SLE) is an autoimmune disease resulting from a loss of tolerance to multiple self antigens, and characterized by autoantibody production and inflammatory cell infiltration in target organs, such as the kidneys and brain. T cells are critical players in SLE pathophysiology as they regulate B cell responses and also infiltrate target tissues, leading to tissue damage. Abnormal signaling events link to defective gene transcription and altered cytokine production, contributing to the aberrant phenotype of T cells in SLE. Study of signaling and gene transcription abnormalities in SLET cells has led to the identification of novel targets for therapy.
\end{abstract}

\section{Introduction: T cells and systemic lupus erythematosus}

Systemic lupus erythematosus (SLE) is an autoimmune disease that afflicts mainly women in the reproductive years. It is a multisystem disease affecting the joints, skin, kidneys and brain and is characterized by autoantibody production by dysregulated B cells, target organ infiltration by inflammatory $\mathrm{T}$ cells and aberrant immune cell activation due to abnormal antigen presenting cell (APC) function. While aberrant $\mathrm{T}$ cells provide help to autoreactive B cells, they also infiltrate target organs, causing damage, and thus are key players in SLE disease pathogenesis. Understanding the underlying defects within $\mathrm{T}$ lymphocytes is of utmost importance not only for understanding disease pathophysiology, but also for identifying predictive biomarkers and better therapeutic targets. T lymphocytes from SLE patients are unique in that they resemble naïve or somewhat anergic $\mathrm{T}$ cells in certain ways, such as their reduced ability to produce cytokines like interferon- $\gamma$ and IL2, but simultaneously

*Correspondence: vmoulton@bidmc.harvard.edu

Division of Rheumatology, Department of Medicine, Beth Israel Deaconess Medical Center, Harvard Medical School, Boston, MA 02115, USA bear characteristics reminiscent of activated/memory $\mathrm{T}$ cells, such as the overall increased tyrosine phosphorylation of signaling intermediates, accelerated calcium flux responses, altered expression of signaling subunits such as the $\mathrm{T}$ cell receptor (TCR) zeta and FcR $\gamma$, and expression of adhesion or costimulatory molecules such as CD44 and CD40L. The following sections describe in detail these and other $\mathrm{T}$ cell signaling abnormalities that are responsible for their defective phenotype and function and may potentially contribute to disease pathogenesis.

\section{Early signaling events \\ Lipid rafts}

Lipid rafts are sphingolipid-cholesterol-GM1-rich microdomains bearing TCR-CD3 complexes and associated signaling molecules distributed on the $\mathrm{T}$ cell surface. In normal T cells, TCR stimulation leads to clustering of these rafts to aid formation of the immunological synapse, allowing for cognate interactions with corresponding molecules on APCs. Freshly isolated SLE T cells, however, display pre-clustered lipid rafts, indicating that the $\mathrm{T}$ cells are 'poised' for activation. In addition, these lipid rafts contain an altered composition of residing molecules on their surface. Alterations include the increased expression of FcR $\gamma$, Syk, and phospholipase $\mathrm{C}$ (PLC) $\gamma$, with decreased expression of the lymphocyte kinase Lck. The localization of tyrosine phosphatase CD45 within the lipid rafts and its association with and activation of Lck are abnormal, leading to the degradation and thus reduced expression of Lck [1-4]. The costimulatory molecule cytotoxic $\mathrm{T}$ lymphocyte associated antigen 4 (CTLA4), a signaling component of the lipid raft, is an important negative regulator of TCR activation. Expression of CTLA4 is found to be increased in freshly isolated T cells from SLE patients [5]; paradoxically, however, it is unable to control the aberrant $\mathrm{T}$ cell activation. Blocking the CTLA4-B7 signaling pathway appears to impede disease progression in animal models of lupus, although timing of treatment is important, such that early treatment prevents or ameliorates disease $[6,7]$. Continuous exposure of $\mathrm{T}$ cells to autoantigen and/or circulating anti-CD3/TCR autoantibodies [8] may account for the observed aggregated lipid rafts on freshly 
isolated $\mathrm{T}$ cells from the peripheral blood of SLE patients. The pre-aggregated lipid rafts contribute to the pathogenesis of SLE, as evidenced in the lupus-prone MRL/lpr mouse. In this mouse, the percentage of T cells with clustered lipid rafts increases with age and peaks before lupus pathology development. More importantly, acceleration of lipid raft aggregation leads to disease advancement, whereas disruption of the aggregates delays pathology [9]. Ex vivo treatment of T lymphocytes from SLE patients with atorvastatin, an inhibitor of 3-hyroxy-3-methylgluteryl CoA reductase that disrupts lipid rafts, showed reduced co-localization of CD45 and Lck, thus reducing the active form of Lck within the rafts. Furthermore, TCR activation not only restored the ERK phosphorylation but also decreased their production of the cytokines IL6 and IL10, which are implicated in SLE pathogenesis. These results show that statins may have therapeutic value in restoring signaling defects in SLE T cells and potentially disease [10].

\section{TCR-CD3 complex}

The TCR is the surface sensor for antigens presented to lymphocytes in the context of the MHC molecule by APCs. The TCR $\alpha$ and $\beta$ chains are closely coupled to the CD3 $\delta, \varepsilon, \gamma$, and $\zeta$ chains to form the TCR-CD3 complex. Each subunit of the $\zeta$ chain bears three immunoreceptor tyrosine activation motifs (ITAMs); thus, the $\zeta \zeta$ homodimer bears a total of six ITAMs and is a critical signaling transducer of $\mathrm{T}$ cells. In naïve $\mathrm{T}$ cells, antigen recognition brings together the TCR, the co-receptor molecule (CD4 or CD8) and the tyrosine phosphatase CD45 on the T cell surface within cholesterol-rich domains called lipid rafts. CD45 removes inhibitory phosphates from the Src family lymphocyte kinase (Lck), and the CD3 chain is phosphorylated at the six ITAMs by Lck. The $\mathrm{CD} 3 \zeta$ chain then recruits the zeta associated protein of $70 \mathrm{kDa}$ (ZAP70) kinase, which is also phosphorylated by Lck. ZAP70 then phosphorylates the adaptor proteins Linker of activation in T cells (LAT) and SLP-76, thus transmitting the signal downstream into three distinct pathways. The adaptor proteins bind and activate the enzyme PLC $\gamma$ on one hand and activate the Ras-mitogen-activated protein kinase (MAPK) pathway through guanine nucleotide exchange factors on the other. PLCY cleaves phosphatidylinositol bisphosphate into diacyl glycerol and inositol trisphosphate. Diacyl glycerol activates protein kinase C (PKC), which activates the transcription factor NF- $\mathrm{kB}$. Inositol trisphosphate leads to opening of the calcium channels, increased intracellular calcium concentrations and activation of the phosphatase calcineurin, which dephosphorylates and activates the transcription factor Nuclear factor of activated T cells (NFAT). Finally, the Ras-MAPK cascade induces and activates fos protein, a component of the transcription factor Activated protein 1 (AP1).
Activation of NF- $\mathrm{kB}$, NFAT and AP1 leads to nuclear translocation of these factors and activation of target gene transcription, cell proliferation and differentiation [11].

Triggering of the TCR in SLE T cells leads to an abnormally accelerated and heightened tyrosine phosphorylation of signaling intermediates, and increased calcium flux characterizing their hyper-responsive phenotype [12]. The stronger signaling is evidenced by the earlier and greater overall tyrosine phosphorylation of signaling intermediates. SLE T cells display a unique rewiring of the surface TCR-CD3 complex wherein expression of the $\mathrm{CD} 3 \zeta$ chain is decreased in cells from a

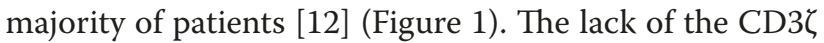
chain in the TCR-CD3 complex is structurally and functionally replaced by the homologous Fc receptor gamma ( $\mathrm{FcR}_{\mathrm{c}}$ ) chain [13]. FcR $\gamma$ was initially identified as the Fc portion of the IgE receptor in mast cells and has structural and functional similarity to the $\zeta$ chain, although the $\mathrm{CD} 3 \zeta$ chain has three ITAMs whereas FcR $\gamma$ has only one. Upon stimulation of SLE T cells, the FcR $\gamma$ chain recruits the spleen tyrosine kinase (Syk) instead of the normally recruited ZAP70. The FcR $\gamma$-Syk interaction is exponentially (>100-fold) stronger than that of the $\zeta$ chain-ZAP 70 combination, rendering a stronger downstream intracellular signal [14]. While this leads to abnormally increased calcium influx, it does not translate into higher IL2-producing capacity of these cells. Rather, the SLE T cells are poor producers of IL2, rendering their somewhat 'anergic' phenotype. Interestingly, replenishment of the CD3 $\zeta$ chain in SLE T cells in vitro normalizes the intracellular calcium flux and more importantly restores IL2 production [15], thus suggesting a key role for the $\mathrm{CD} 3 \zeta$ chain in the $\mathrm{T}$ cell defect. Thus, correction of a missing signaling molecule in SLE T cells may result in normalization of effector $\mathrm{T}$ cell function.

The decreased expression of the CD3 $\zeta$ chain in SLE T cells has been attributed to defects at multiple levels, including defective gene transcription [16], aberrant mRNA splicing [17], poor transcript stability of alternative splice variants [18], and increased protein degradation by caspase-mediated [19], ubiquitin-proteasomemediated and lysosomal-mediated mechanisms [20]. The transcriptional activity of the $C D 3 \zeta$ promoter is limited because of limited binding of the transcriptional enhancer E-74-like-factor (Elf)-1 and increased binding of the repressor c-AMP response element modulator (CREM) $\alpha$ [21], which is increased in SLE T cells. The CD3 $\zeta$ mRNA in SLE $\mathrm{T}$ cells is produced in many alternatively spliced forms lacking coding regions that may result in non-functional or unstable isoforms [17]. The $\mathrm{CD} 3 \zeta$ chain protein is degraded by ubiquitin-mediated proteolysis [22], lysosomal degradation [20] as well as by caspase 3, which is expressed at increased levels in SLE T cells [19]. Because replenishment of $\mathrm{CD} 3 \zeta$ results in 


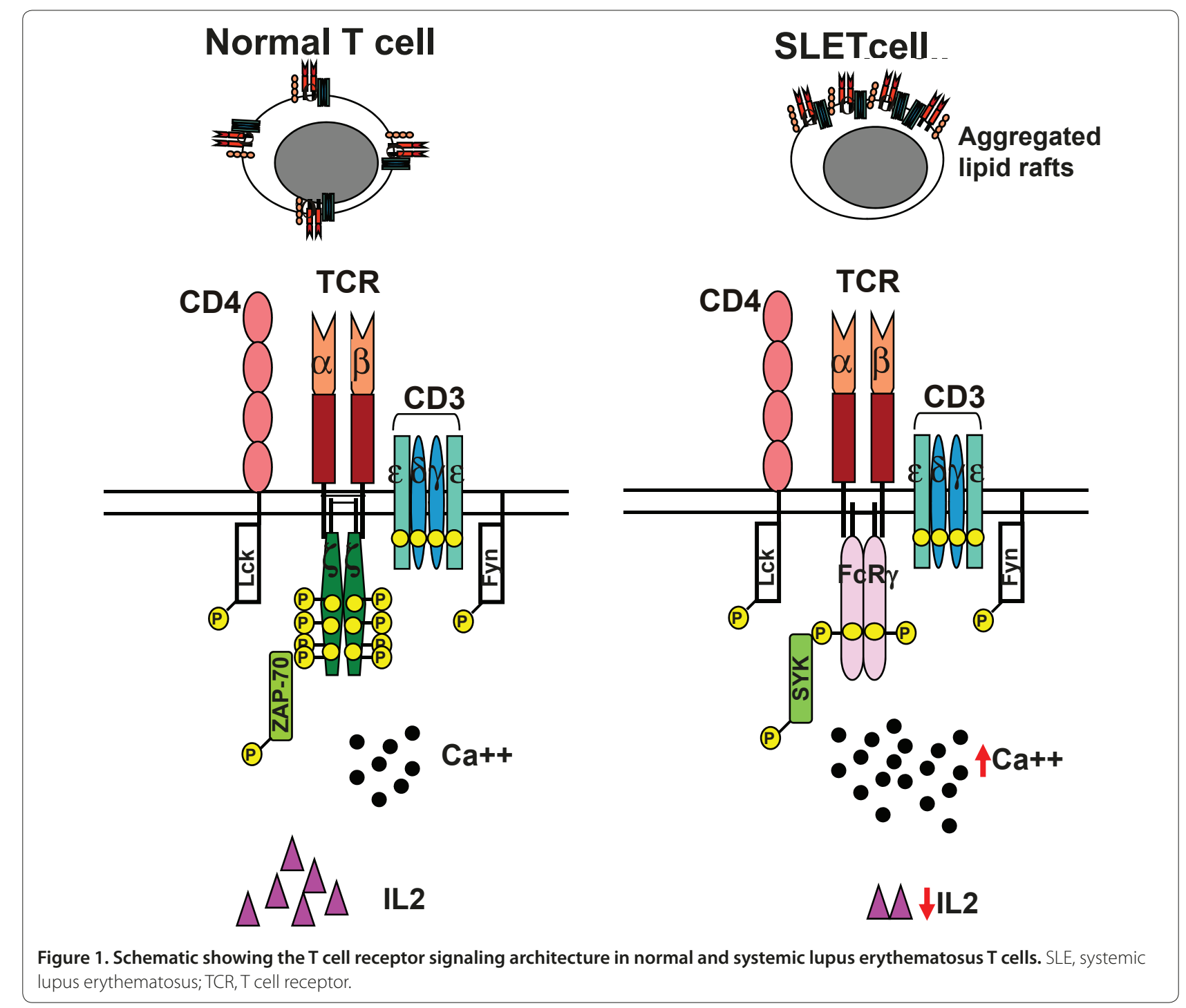

increased production of IL2, understanding the molecular mechanisms that lead to its decreased production has allowed the proposition of interventions that are expected to lead to normalized $\mathrm{T}$ cell function. For example, inhibition of caspase 3 [19], blocking of mammalian target of rapamycin (mTOR) with rapamycin [23] and silencing of the transcriptional repressor CREM $\alpha$ [21] may be considered as therapeutic tools in SLE.

\section{Kinases}

The restructuring of the SLE TCR is characterized by abnormally high expression of the Syk kinase. Normally, TCR stimulation leads to the recruitment of ZAP70

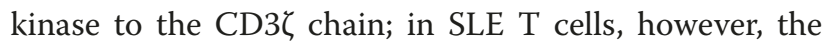
substituted FcR $\gamma$ recruits Syk kinase. The FcR $\gamma$-Syk interaction is significantly stronger than that of the zetaZAP 70 association, and contributes to the stronger downstream signaling as evidenced by hyper-phosphorylation of intermediate signaling molecules and increased calcium flux in SLE T cells. The increased expression and activity of Syk in SLE T cells is evidenced by its increased expression in the lipid rafts and increased association with downstream molecules involved in actin polymerization and calcium signaling, namely Vav-1 and PLC 1 [24]. Accordingly, Syk inhibition with the pharmacological agent R406 led to retardation of actin polymerization kinetics in SLE T cells. Inhibition of Syk using the R788 inhibitor not only suppresses the development of skin and kidney disease but also abolishes established disease in lupus-prone mice [25]. Therefore, Syk inhibition is under consideration for clinical trials in patients with SLE.

Phosphorylated PKB content is clearly increased in MRL-lpr CD4+ cells compared to control CD4+ cells and 
it was considered a proper therapeutic target. Indeed, inhibition of phosphoinositide 3-kinase (PI3K) $\gamma$ by the compound AS605240 led to significantly reduced severity of glomerulonephritis prolonged survival in lupus-prone MRL/lpr mice [26]. Should PI3K $\gamma$ levels be found to be increased in human SLE T cells, it should also be considered for therapeutic targeting.

\section{Adhesion/co-stimulation}

\section{CD44, phosphorylated ezrin/radixin/moiesin}

CD44 is a T cell surface adhesion molecule that recognizes and binds to its ligand hyaluronic acid in tissues and thus enables $\mathrm{T}$ cells to migrate into peripheral tissues. Naïve T cells express low levels of CD44 whereas activated and memory $\mathrm{T}$ cells express high amounts of this membrane receptor. The CD44 gene undergoes extensive alternative splicing of its variable exons, leading to the generation of numerous alternatively spliced isoforms. T cells from SLE patients express high amounts of certain CD44 isoforms (v3 and v6) and their expression correlates with a patient's disease activity [27]. Renal biopsy of patients with lupus nephritis revealed $\mathrm{T}$ cells from kidneys to express CD44, and also showed phosphorylated ezrin/radixin/moiesin (pERM) proteins to be involved in the CD44 signaling cascade [28], suggesting that expression of these homing molecules may allow the $\mathrm{T}$ cells to migrate abnormally into the kidneys. pERM is responsible for the increased polarization, adhesion and migration of T cells in SLE patients, as evidenced by the presence of pERM+ T cells in kidney infiltrates. In addition, CD44 requires pERM in order to acquire adhesive capacity. ERM is phosphorylated by the rho-associated protein kinase (ROCK) and its inhibition makes SLE $\mathrm{T}$ cells unable to adhere to hemagglutinin-coated membranes [29]. The finding of CD44+pERM+ cells in both peripheral blood and diseased kidneys of SLE patients suggests that $\mathrm{T}$ cells become activated and acquire pathogenic potential while in the periphery and then migrate to target tissues and lead to pathology. More recently, ROCK was found necessary for the production of IL17, and treatment of lupus-prone mice with a ROCK inhibitor led to improved disease [30]. Therefore, the ex vivo human studies and the preclinical data strongly urge the consideration of ROCK inhibitors in the treatment of SLE patients.

\section{Signaling lymphocytic activation molecule family}

The signaling lymphocytic activation molecule (SLAM) family comprises nine transmembrane signaling proteins and is a subtype of the immunoglobulin superfamily. These proteins serve as co-stimulatory molecules on the surface of $\mathrm{T}$ cells and are involved in lineage commitment during hematopoiesis, $\mathrm{T}$ cell function as well as B cell activation and natural killer cell inhibition. Most have a unique tyrosine-based switch motif that has high affinity for the SH2 domain-bearing molecules SLAM-associated protein (SAP) and EAT2. Genome-wide association studies in SLE patients' families have shown the presence of a susceptibility locus on chromosome 1q23 that also includes the SLAM genes [31]. A recent study found defects within the SLAMF such that co-engagement of SLAMF3 or 6 with CD3 in human SLE T cells failed to restore IL2 production [32]. Further understanding of the role of the SLAM family molecules in human SLE will reveal their role in disease and potential use in therapy.

\section{CD40 ligand/inducible T cell co-stimulator}

Activated $\mathrm{T}$ cells express CD40 ligand (CD40L) and provide cognate help to $\mathrm{CD} 40$-expressing $\mathrm{B}$ cells via the CD40-CD40L interaction. SLE T cells not only show increased and prolonged expression of CD40L upon activation but also exhibit increased levels of baseline CD40L, which correlated with disease activity in some patients [33,34]. Reciprocally, hyperactive B cells can stimulate T cells, which upregulate CD40L. Dysregulated $\mathrm{T}$ cells then provide help to autoreactive B cells, inducing the production of autoantibodies. The increased expression of CD40 in the kidneys in SLE patients was shown to correlate with the presence of CD40L-expressing peripheral blood mononuclear cells. Preliminary clinical trials using two different anti-CD40L antibodies, although showing promising results [35], led to severe unforeseen adverse effects [36]. Despite interesting preclinical data on the therapeutic potential of the disruption of the CD40-CD40L interaction, clinical efforts so far have not met the predictions.

Another co-stimulatory molecule - inducible T cell costimulator (ICOS) - is required for controlling the local inflammatory effector functions of $\mathrm{T}$ cells infiltrating the kidneys in MRL/lpr lupus-prone mice [37]. ICOS is also needed for IL21 secretion by extrafollicular helper T cells as well as plasma cell differentiation and IgG production in chronic autoimmunity evidenced in the lupus-prone mice lacking ICOS [38]. These studies suggest the potential importance of ICOS blockade as a therapeutic measure for autoimmune disease.

\section{Intermediate signaling events MAPK signaling pathway}

Abnormalities in the MAPK signaling pathway have been reported in SLE T cells and include an impaired extracellular signal-regulated kinase (ERK) signaling cascade. The ERK signaling is diminished in SLE T cells [39]. Ras activation is shown to be abnormal in SLE patients [39], and altered Ras guanyl nucleotide releasing protein 1 (RasGRP1) and PKC $\delta$ activation are linked to this defect $[40,41]$. Defective PKC $\delta$ activation leads to abnormal ERK pathway signaling, resulting in DNA hypomethylation, 
which seemingly contributes to the development of SLE [42]. Decreased phosphorylation of PKC 8 , ERK, MEK and Raf affects DNA methylation of target genes by leading to decreased expression of the DNA methyl transferase (DNMT). Accordingly, $\mathrm{T}$ cells from SLE patients have reduced levels of the enzyme DNMT1. While the exact link between the defective MAPK signaling and autoimmunity is incompletely understood, two important consequences are the effect on DNA methylation and the effect on reduced c-fos expression, which is a component of the AP1 transcription factor.

\section{mTOR}

Production of reactive oxygen intermediates and ATP synthesis are critical determinants of $\mathrm{T}$ cell activation, proliferation, cytokine production and cell death. Reactive oxygen intermediate and ATP generation are tightly regulated by the mitochondrial transmembrane potential. Persistent mitochondrial hyperpolarization, increased reactive oxygen intermediate production and ATP depletion in SLE T cells are responsible for their increased spontaneous and decreased activation-induced apoptosis. mTOR, a serine threonine kinase member of the PI3K-related kinase family, is a sensor of the mitochondrial transmembrane potential and is increased in SLE T cells [20]. Furthermore, nitric oxide-induced mTOR activation leads to lysosomal degradation of the

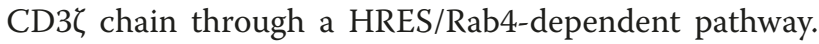
HRES/Rab4 is a small GTPase that regulates endocytic recycling of surface receptors by the early endosomes [20]. mTOR inhibition in patients with SLE resulted in clinical improvement [23], and therefore a proper trial is warranted.

\section{Gene transcription CD3//FcR $y$ transcription}

Elf-1 is a member of the Ets family of transcription factors and is shown to bind and activate transcription of the $C D 3 \zeta$ gene. Examination of ELF-1 in SLE patients showed two subsets of patients - one that expressed reduced amounts of the $98-\mathrm{kDa}$ DNA binding form of the ELF-1 protein and another that showed reduced binding capacity to the $C D 3 \zeta$ promoter [16]. In addition, protein phosphatase (PP)2A dephosphorylates Elf-1 at Thr231, resulting in limited expression and binding activity of the $98-\mathrm{kDa}$ form. Thus, the lack of a functional Elf- 1 accounts for the reduced CD3 3 transcription in SLE $\mathrm{T}$ cells. Normal $\mathrm{T}$ cells when stimulated generate effectors that downregulate $\mathrm{CD} 3 \zeta$ and concomitantly upregulate $\mathrm{FcR} \gamma$ in the TCR complex, thus functionally replacing the $\mathrm{CD} 3 \zeta$ chain [43]. In SLE $\mathrm{T}$ cells, reconstitution of the $\mathrm{CD} 3 \zeta$ chain reciprocally leads to downregulation of the $\mathrm{FcR} \gamma$ and restoration of calcium flux and IL2 production [15]. Interestingly, Elf-1 was also shown to bind GGAA elements in the FcR $\gamma$ promoter and suppress FcR $\gamma$ expression [44], indicating that Elf-1 may act as a molecular switch in the reciprocal regulation of $\mathrm{CD} 3 \zeta$ and FcR $\gamma$ in SLE T cells. The reduced expression of Elf- 1 in SLE T cells may partly explain the increased expression of $\mathrm{FcR} \gamma$ concomitant with the reduced expression of $\mathrm{CD} 3 \zeta$.

\section{IL2 transcription}

TCR triggering induces intracellular signaling cascades, ultimately leading to gene transcription. In addition to the aberrations in signaling within SLE T cells a number of defects in the expression and/or function of transcription factors are observed in SLE T cells. SLE T cells are poor producers of the vital growth and proliferationinducing cytokine IL2. Defective transcription is an important factor of this deficiency. NF- $\mathrm{kB}$, NFAT, AP1, CREB (cAMP response element-binding) and CREM are transcription factors involved in IL2 transcription (Figure 2). $\mathrm{NF}-\mathrm{kB}$ is a heterodimer of the p65/p50 subunits and the expression of the p65 subunit is decreased in SLE T cells [45].

The AP1 family of transcription factors is formed by heterodimers and homodimers of fos ( $v$-fos, c-fos, fosB, fra1, and fra2) and jun (v-jun, c-jun, jun-b, jun-d) proteins [46]. Upon antigenic stimulation, the jun and fos proteins are expressed, and AP1 (especially the c-fos/cjun heterodimers) binds to the IL2 promoter. Decreased c-fos expression is responsible for reduced AP1 binding activity to the IL2 promoter in SLE T cells [47].

An imbalance between the transcription factors CREB and CREM $\alpha$ plays an important role in the regulation of IL2 production in SLE T cells. The CREM gene undergoes alternative splicing to produce many isoforms, some of which are transcriptional activators, and others repressors such as CREM $\alpha$. Both activated CREB and CREM $\alpha$ reciprocally bind to a CRE site at position -180 on the IL2 promoter. Active (phosphorylated) CREB is a transcriptional activator of IL2 while phosphorylated CREM $\alpha$ is a transcriptional repressor of the IL2 gene. Reduced production of IL2 by SLE T cells is regulated in part by the increased expression and activity of CREM $\alpha$. Decreased protein kinase A activity leads to reduced phosphorylation of CREB [48], thus reducing the availability of phosphorylated CREB for binding the IL2 promoter. Additionally, abnormally increased expression of the PP2A enzyme, which dephosphorylates CREB, leads to reduced availability of phosphorylated CREB for binding to the IL2 promoter [49]. CREM $\alpha$ is phosphorylated by a number of kinases, including the calcium/ calmodulin-dependent kinase IV (CAMKIV). Increased expression of CAMKIV is observed in the nucleus of SLE $\mathrm{T}$ cells. Treatment of normal T cells with SLE serum, which presumably leads to triggering of the TCR by 


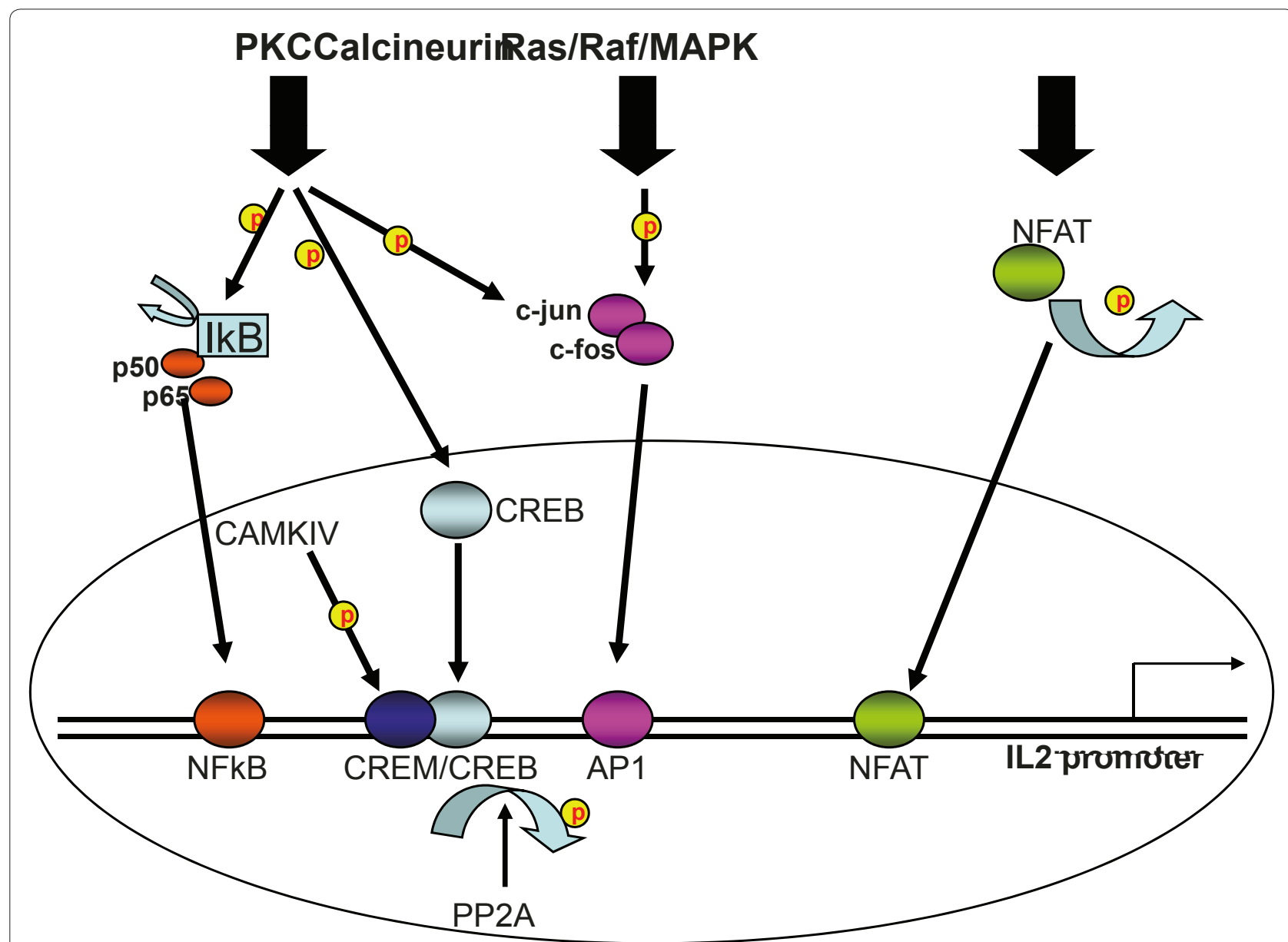

Figure 2. Schematic showing transcription factors involved in IL2 production in T cells. AP1, activated protein 1; CAMKIV, calcium/calmodulindependent kinase IV; CREB, CAMP response element-binding; CREM, CAMP response element modulator; MAPK, mitogen-activated protein kinase; NFAT, nuclear factor of activated T cells; PKC, protein kinase C; PP, protein phosphatase.

anti-CD3 autoantibodies in SLE serum, leads to the activation and nuclear translocation of CAMKIV and increased complex formation at the -180 site of the IL2 promoter [8]. CAMKIV is increased also in T cells from the MRL/lpr lupus-prone mouse. Administration of a CAMKIV inhibitor to these mice was able to prevent and even correct disease pathology [50].

NFAT binds to promoters of the genes encoding IL2 and CD40L and activates their transcription [51], and NFAT expression is abnormally high in SLE $\mathrm{T}$ cells. While this accounts for increased expression of CD40L, it does not promote increased IL2 production. The reason for this discrepancy is that while NFAT can alone bind to and activate the CD40L promoter, binding to the IL2 promoter requires AP1 binding to adjacent sites. Defective AP1 activity thus hampers the NFAT action on IL2 transcription. In resting $\mathrm{T}$ cells, NFAT is phosphorylated and inactive in the cytoplasm. Upon $\mathrm{T}$ cell stimulation, dephosphorylation by the calcium responsive calcineurin phosphatase, NFAT translocates into the nucleus and activates gene transcription. In SLE T cells, the increased calcium flux with resultant increased calcineurin expression leads to increased dephosphorylation of NFAT and thus increased availability inside the nucleus and aberrant target gene expression.

\section{IL17 transcription}

IL17 has recently emerged as a key inflammatory cytokine, playing a central role in the pathogenesis of several autoimmune diseases, including SLE [52]. Serum IL17 levels are increased in patients with SLE [53] and the frequency of IL17-producing $\mathrm{T}$ cells is increased in peripheral blood of patients with SLE [54]. An expanded population of CD3+CD4-CD8- double negative T cells was shown to produce increased amounts of IL17 in SLE patients. Furthermore, $\mathrm{T}$ cell infiltrates in the kidneys were composed of double negative and IL17-producing $\mathrm{T}$ cells in patients with lupus nephritis [54]. Differentiation 


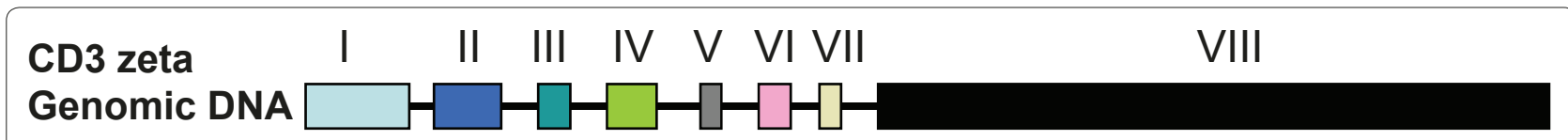
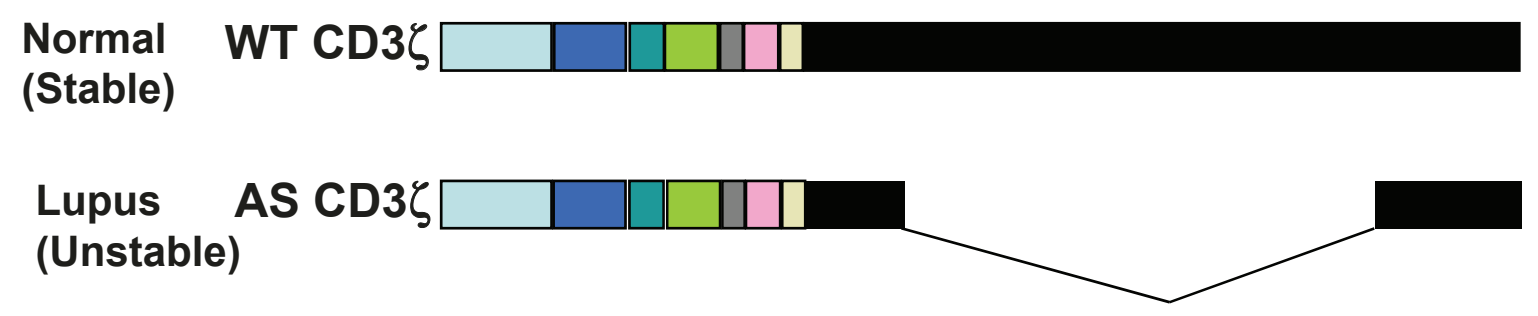

Figure 3. Schematic showing the $C D 3 \zeta$ gene. Genomic DNA with eight exons (top), the mRNA with a full-length 906-bp 3'UTR (WT; middle) and the 344-bp alternatively spliced (AS) 3' UTR variant (bottom). SLET cells express increased amounts of the unstable AS splice variant relative to the stable WT isoform.

of CD4 T cells into IL17-producing Th17 cells requires the presence of the inflammatory cytokines IL6, IL23, IL21 and transforming growth factor- $\beta$, although human memory $\mathrm{T}$ cells are capable of producing IL17 with only CD3 CD28 priming [55,56]. IL23 is required to drive this differentiation, while IL21 sustains and is required for maintenance of IL17 production. IL6, IL21 and IL23 all activate STAT3, which can bind and activate the IL17 and IL21 genes directly [57]. The expression and activity of STAT3 is increased in SLE T cells and is responsible in part for the enhanced chemokine-mediated migration of these cells [58]. The $I L 17$ gene transcription is regulated by the retinoid related orphan receptor ROR $\gamma \mathrm{t}$ and ROR $\alpha$ transcription factors. ROR $\gamma$ t is expressed exclusively in Th17 cells and is necessary for IL17 production [59]. Aside from the pro-inflammatory effects mediated directly by IL17, it can also contribute to pathogenesis through its effect on other cell types. High levels of antidsDNA IgG and IL6 were produced by peripheral blood mononuclear cells from patients with lupus nephritis when cultured with IL17 [60], suggesting its role in B cell activation. Genetic disruption of the IL23 receptor in the lupus prone B6.lpr mouse results in reduction of the numbers of double negative cells, reduced IL17 production, and improved renal pathology. Similarly, blockade of IL23 with an anti-IL23 antibody improved disease manifestations [61]; therefore, disruption of the path from IL23 to IL17 may be of clinical value.

\section{Alternative splicing in systemic lupus erythematosus}

$\mathrm{T}$ cells from patients with SLE display abnormal alternative splicing of a number of genes involved in diverse functions, such as signaling, homing and transcription regulation. Examples include the signaling molecule $\mathrm{CD} 3 \zeta$, adhesion molecule $\mathrm{CD} 44$ and the transcription

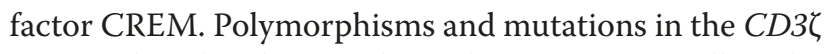
gene within the 5' UTR, the coding region as well as the 3' UTR have been reported. Notably, a 3' UTR splice variant with reduced mRNA stability is expressed in increased amounts in SLE T cells (Figure 3) [18], likely due to the reduced expression of the serine arginine splicing factor ASF/SF2, which has been shown to repress the generation of this unstable isoform [62]. The CREM gene undergoes splicing to produce distinct isoforms with opposing roles in transcription regulation - some being transcriptional activators, such as CREMtau2 $\alpha$, while others repress transcription, such as CREM $\alpha$ and inducible cAMP early repressor ICER. Increased expression and activity of CREM $\alpha$ contributes to the defective IL2 transcription in SLE T cells [63]. Alternative splicing of CD44 leading to the expression of CD44v3 and CD44v6 in SLE T cells was discussed above. Understanding the regulation of alternative splicing of these molecules in SLE T cells may lead to identification of potential therapeutic targets.

\section{Epigenetics}

DNA methylation leads to chromatin inactivation and suppression of gene expression whereas hypomethylation of DNA regulatory elements activates gene expression. Hypomethylation is a characteristic of several genes involved in SLE T cell pathophysiology and contributes to the overexpression of genes responsible in lupus pathogenesis and disease development [64]. Typical examples of genes that are involved in the pathogenesis of SLE and have been found hypomethylated include CD11a, perforin, CD70 and CD40L [42]. Hypomethylation of the $P P 2 A$ promoter is a contributing factor responsible for the overexpression of this enzyme in SLE T cells [65]. Expression and activity of the DNMT enzyme, responsible for DNA methylation, were reduced in $\mathrm{T}$ cells from 
active SLE patients compared to healthy donors [66]. Recent evidence shows the role of the growth arrest and DNA damage-induced (GADD) 45alpha gene in promoting lupus-like autoimmunity by inducing gene hypomethylation in CD4+ T cells from SLE patients [67].

\section{Conclusion}

While there is a wide range of anomalies within SLE $\mathrm{T}$ cells, certain common themes emerge and provide clues to central molecular mechanisms linking these various defects. These include chronic activation, epigenetic mechanisms, such as defective DNA methylation, and aberrant gene regulation, such as defective alternative splicing. Several defects observed in the SLE TCR signaling pathway are suggestive of an activation state and may be due to activation by APC and/or autoantibodies. For example, activation induces changes in

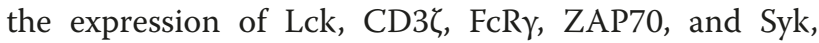
phosphorylation of intermediates, and calcium fluxing, many of which are also observed in SLE T cells. However, SLE T cells concurrently exhibit unique features that do not occur in normal activated $\mathrm{T}$ cells. For example, while the activation of normal $\mathrm{T}$ cells induces downregulation of the $\zeta$ chain, there is no downregulation of the mRNA this is not observed in SLE T cells wherein the transcription of the $\zeta$ chain is defective due to specific aberrations, such as reduced activity of the transcriptional enhancer Elf-1 and others as explained in the sections above. Aberrant DNA methylation affects a number of genes in SLE patients, such as those encoding CD40L, CD70, CD11a, and PP2A. Aberrant alternative splicing of many genes $(C D 3 \zeta, C D 44, C R E M)$ is observed in SLE $\mathrm{T}$ cells and may reflect a global deregulation of this process, which may be of genetic origin or may reflect defects in the cellular microenvironment.

In summary, $\mathrm{T}$ cells from SLE patients have several biochemical abnormalities endowing them with a hyperexcitable phenotype but a defective gene transcription program. These result in a peculiar cell type with properties of activated/effector cells on the one hand but a somewhat anergic state on the other. Many molecules involved in the development of this phenotype have been identified and should eventually lead to better understanding and management of this complex disease. SLE is a heterogeneous disease, and it is likely that several molecular defects result in the same/similar clinical outcomes. It would be interesting and important to identify links between these signaling defects and clinical profiles of SLE patients. Simultaneous study of all abnormally expressed genes may provide additional insights into the identification of subgroups among patients with SLE who share common biochemical aberrations. Identification of such groups of patients may lead to the suggestion of specific treatment(s) able to correct defined abnormalities.
Our laboratory has initiated such an approach [68] and preliminary data along these lines are promising.

The elucidation of aberrant signaling and gene transcription in T cells from SLE patients is important, as this will lead to the identification of novel drug targets, gene therapeutic measures, and, importantly, disease-predicting biomarkers. In this review, we have discussed the signaling and gene transcription aberrations in T lymphocytes, and pointed out targets that can be exploited therapeutically. We have paid attention to abnormalities first detected in human SLE $\mathrm{T}$ cells and then validated in lupus-prone mice using drug inhibitors or genetic manipulations. Syk, ROCK and CAMKIV inhibition as discussed herein deserve proper clinical consideration. In addition, inhibition of the IL23-IL17 axis deserves consideration for clinical trials using either anti-IL17 or IL23 antibodies or a decoy IL23 receptor. While B cell depletion has benefited a number of SLE patients, a clinical trial on $B$ cell depletion therapy has yielded negative results and some biologics such as anti-Blys therapy have had mild effects. This should direct our attention also to the development of therapeutic targets that correct $\mathrm{T}$ cell function. A subset of patients may respond well to B-celldirected therapy, whereas another subset to T-cellmodifying approaches. The extremely complex nature of the disease, with heterogeneity not only at the clinical level but also at the molecular level, suggests the need for a case-by-case treatment modality rather than a blanket approach.

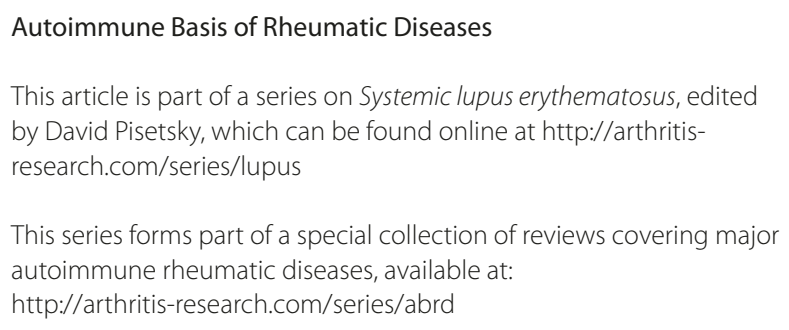

This series forms part of a special collection of reviews covering major autoimmune rheumatic diseases, available at:

http://arthritis-research.com/series/abrd

\section{Abbreviations}

AP1, activated protein 1; APC, antigen presenting cell; CAMKIV, calcium calmodulin-dependent kinase IV; CD40L, CD40 ligand; CREB, CAMP response element-binding; CREM, CAMP response element modulator; DNMT, DNA methyl transferase; ERK, extracellular signal-regulated kinase; ICOS, inducible T cell co-stimulator; IL, interleukin; ITAM, immunoreceptor tyrosine activation motif; MAPK, mitogen-activated protein kinase; mTOR, mammalian target of rapamycin; NF, nuclear factor; NFAT, nuclear factor of activated T cells; pERM, phosphorylated ezrin/radixin/moiesin; PI3K, phosphoinositide 3-kinase; PKC, protein kinase C; PLC, phospholipase C; PP, protein phosphatase; ROCK, rhoassociated protein kinase; SLAM, signaling lymphocytic activation molecule; SLE, systemic lupus erythematosus; TCR, T cell receptor; UTR, untranslated region.

\section{Competing interests}

The Tsokos lab has received a research grant to study the role of a Syk inhibitor from Rigel pharmaceuticals, San Francisco, CA. 


\section{Acknowledgements}

We would like to thank Dr Vasileios Kyttaris for useful insights on the manuscript.

\section{Published: 17 March 2011}

\section{References}

1. Jury EC, Kabouridis PS, Abba A, Mageed RA, Isenberg DA: Increased ubiquitination and reduced expression of LCK in T lymphocytes from patients with systemic lupus erythematosus. Arthritis Rheum 2003, 48:1343-1354

2. Jury EC, Kabouridis PS, Flores-Borja F, Mageed RA, Isenberg DA: Altered lipid raft-associated signaling and ganglioside expression in $T$ lymphocytes from patients with systemic lupus erythematosus. J Clin Invest 2004 113:1176-1187.

3. Krishnan S, Nambiar MP, Warke VG, Fisher CU, Mitchell J, Delaney N, Tsokos GC: Alterations in lipid raft composition and dynamics contribute to abnormal T cell responses in systemic lupus erythematosus. J Immunol 2004, 172:7821-7831.

4. Kabouridis PS, Jury EC: Lipid rafts and T-lymphocyte function: implications for autoimmunity. FEBS Lett 2008, 582:3711-3718.

5. Liu MF, Liu HS, Wang CR, Lei HY: Expression of CTLA-4 molecule in peripheral blood T lymphocytes from patients with systemic lupus erythematosus. J Clin Immunol 1998, 18:392-398.

6. Kristiansen OP, Larsen ZM, Pociot F: CTLA-4 in autoimmune diseases a general susceptibility gene to autoimmunity? Genes Immun 2000 1:170-184.

7. Salomon B, Bluestone JA: Complexities of CD28/B7: CTLA-4 costimulatory pathways in autoimmunity and transplantation. Annu Rev Immuno/ 2001, 19:225-252

8. Juang YT, Wang Y, Solomou EE, Li Y, Mawrin C, Tenbrock K, Kyttaris VC, Tsokos GC: Systemic lupus erythematosus serum IgG increases CREM binding to the IL-2 promoter and suppresses IL-2 production through CaMKIV. J Clin Invest 2005, 115:996-1005.

9. Deng GM, Tsokos GC: Cholera toxin B accelerates disease progression in lupus-prone mice by promoting lipid raft aggregation. J Immuno/ 2008, 181:4019-4026.

10. Jury EC, Isenberg DA, Mauri C, Ehrenstein MR: Atorvastatin restores Lck expression and lipid raft-associated signaling in T cells from patients with systemic lupus erythematosus. J Immuno/ 2006, 177:7416-7422.

11. Janeway $C A$, Travers $P$, Walport M, Shlomchik M: Signaling through immune system receptors. In Immunobiology: The Immune System in Health and Disease. 5th edition. Edited by Gibbs S. New York: Garland Science; 2001:187-220.

12. Liossis SN, Ding XZ, Dennis GJ, Tsokos GC: Altered pattern of TCR/CD3mediated protein-tyrosyl phosphorylation in T cells from patients with systemic lupus erythematosus. Deficient expression of the T cell receptor zeta chain. J Clin Invest 1998, 101:1448-1457

13. Enyedy EJ, Nambiar MP, Liossis SN, Dennis G, Kammer GM, Tsokos GC: Fc epsilon receptor type I gamma chain replaces the deficient T cell receptor zeta chain in T cells of patients with systemic lupus erythematosus. Arthritis Rheum 2001, 44:1114-1121.

14. Nambiar MP, Fisher CU, Kumar A, Tsokos CG, Warke VG, Tsokos GC: Forced expression of the $\mathrm{Fc}$ receptor gamma-chain renders human $T$ cells hyperresponsive to TCR/CD3 stimulation. J Immuno/ 2003, 170:2871-2876.

15. Nambiar MP, Fisher CU, Warke VG, Krishnan S, Mitchell JP, Delaney N, Tsokos GC: Reconstitution of deficient T cell receptor zeta chain restores $T$ cell signaling and augments T cell receptor/CD3-induced interleukin-2 production in patients with systemic lupus erythematosus. Arthritis Rheum 2003, 48:1948-1955.

16. Juang YT, Tenbrock K, Nambiar MP, Gourley MF, Tsokos GC: Defective production of functional $98-\mathrm{kDa}$ form of Elf- 1 is responsible for the decreased expression of TCR zeta-chain in patients with systemic lupus erythematosus. J Immunol 2002, 169:6048-6055.

17. Nambiar MP, Enyedy EJ, Warke VG, Krishnan S, Dennis G, Kammer GM, Tsokos GC: Polymorphisms/mutations of TCR-zeta-chain promoter and $3^{\prime}$ untranslated region and selective expression of TCR zeta-chain with an alternatively spliced 3 ' untranslated region in patients with systemic lupus erythematosus. J Autoimmun 2001, 16:133-142.

18. Chowdhury B, Tsokos CG, Krishnan S, Robertson J, Fisher CU, Warke RG, Warke VG, Nambiar MP, Tsokos GC: Decreased stability and translation of T cell receptor zeta mRNA with an alternatively spliced 3'-untranslated region contribute to zeta chain down-regulation in patients with systemic lupus erythematosus. J Biol Chem 2005, 280:18959-18966.

19. Krishnan S, Kiang JG, Fisher CU, Nambiar MP, Nguyen HT, Kyttaris VC, Chowdhury B, Rus V, Tsokos GC: Increased caspase-3 expression and activity contribute to reduced CD3zeta expression in systemic lupus erythematosus T cells. J Immuno/ 2005, 175:3417-3423.

20. Fernandez DR, Telarico T, Bonilla E, Li Q, Banerjee S, Middleton FA, Phillips PE, Crow MK, Oess S, Muller-Esterl W, Perl A: Activation of mammalian target of rapamycin controls the loss of TCRzeta in lupus T cells through HRES-1/ Rab4-regulated lysosomal degradation. J Immuno/ 2009, 182:2063-2073.

21. Tenbrock K, Kyttaris VC, AhImann M, Ehrchen JM, Tolnay M, Melkonyan H, Mawrin C, Roth J, Sorg C, Juang YT, Tsokos GC: The cyclic AMP response element modulator regulates transcription of the TCR zeta-chain. J Immunol 2005, 175:5975-5980.

22. Nambiar MP, Enyedy EJ, Fisher CU, Krishnan S, Warke VG, Gilliland WR, Oglesby RJ, Tsokos GC: Abnormal expression of various molecular forms and distribution of $\mathrm{T}$ cell receptor zeta chain in patients with systemic lupus erythematosus. Arthritis Rheum 2002, 46:163-174

23. Fernandez D, Bonilla E, Mirza N, Niland B, Perl A: Rapamycin reduces disease activity and normalizes $T$ cell activation-induced calcium fluxing in patients with systemic lupus erythematosus. Arthritis Rheum 2006 54:2983-2988

24. Krishnan S, Juang YT, Chowdhury B, Magilavy A, Fisher CU, Nguyen H, Nambiar MP, Kyttaris V, Weinstein A, Bahjat R, Pine P, Rus V, Tsokos GC: Differential expression and molecular associations of Syk in systemic lupus erythematosus T cells. J Immunol 2008, 181:8145-8152.

25. Deng GM, Liu L, Bahjat FR, Pine PR, Tsokos GC: Suppression of skin and kidney disease by inhibition of spleen tyrosine kinase in lupus-prone mice. Arthritis Rheum 2010, 62:2086-2092

26. Barber DF, Bartolomé A, Hernandez C, Flores JM, Redondo C, Fernandez-Arias C, Camps M, Rückle T, Schwarz MK, Rodríguez S, Martinez-A C, Balomenos D, Rommel C, Carrera AC: PI3Kgamma inhibition blocks glomerulonephritis and extends lifespan in a mouse model of systemic lupus. Nat Med 2005 11:933-935.

27. Crispín JC, Keenan BT, Finnell MD, Bermas BL, Schur P, Massarotti E, Karlson EW, Fitzgerald LM, Ergin S, Kyttaris VC, Tsokos GC, Costenbader KH: Expression of CD44 variant isoforms CD44v3 and CD44v6 is increased on $T$ cells from patients with systemic lupus erythematosus and is correlated with disease activity. Arthritis Rheum 2010, 62:1431-1437.

28. Cohen RA, Bayliss G, Crispin JC, Kane-Wanger GF, Van Beek CA, Kyttaris VC, Avalos I, Yu CY, Tsokos GC, Stillman IE: T cells and in situ cryoglobulin deposition in the pathogenesis of lupus nephritis. Clin Immunol 2008, 128:1-7.

29. Li Y, Harada T, Juang YT, Kyttaris VC, Wang Y, Zidanic M, Tung K, Tsokos GC: Phosphorylated ERM is responsible for increased T cell polarization, adhesion, and migration in patients with systemic lupus erythematosus. J Immunol 2007, 178:1938-1947.

30. Biswas PS, Gupta S, Chang E, Song L, Stirzaker RA, Liao JK, Bhagat G, Pernis AB: Phosphorylation of IRF4 by ROCK2 regulates IL-17 and IL-21 production and the development of autoimmunity in mice. J Clin Invest 2010, 120:3280-3295.

31. Detre C, Keszei M, Romero X, Tsokos GC, Terhorst C: SLAM family receptors and the SLAM-associated protein (SAP) modulate T cell functions. Semin Immunopathol 2010, 32:157-171.

32. Chatterjee MC, Kis-Toth K, Thai TH, Terhorst C, Tsokos GC: SLAMF6-driven co-stimulation of human peripheral T cells is defective in SLE T cells. Autoimmunity, in press.

33. Koshy M, Berger D, Crow MK: Increased expression of CD40 ligand on systemic lupus erythematosus lymphocytes. J Clin Invest 1996, 98:826-837.

34. Desai-Mehta A, Lu L, Ramsey-Goldman R, Datta SK: Hyperexpression of CD40 ligand by $B$ and T cells in human lupus and its role in pathogenic autoantibody production. J Clin Invest 1996, 97:2063-2073.

35. Boumpas DT, Furie R, Manzi S, Illei GG, Wallace DJ, Balow JE, Vaishnaw A: A short course of BG9588 (anti-CD40 ligand antibody) improves serologic activity and decreases hematuria in patients with proliferative lupus glomerulonephritis. Arthritis Rheum 2003, 48:719-727.

36. Sidiropoulos PI, Boumpas DT: Lessons learned from anti-CD40L treatment in systemic lupus erythematosus patients. Lupus 2004, 13:391-397.

37. Odegard JM, DiPlacido LD, Greenwald L, Kashgarian M, Kono DH, Dong C, Flavell RA, Craft J: ICOS controls effector function but not trafficking 
receptor expression of kidney-infiltrating effector T cells in murine lupus. J Immunol 2009, 182:4076-4084.

38. Odegard JM, Marks BR, DiPlacido LD, Poholek AC, Kono DH, Dong C, Flavell RA, Craft J: ICOS-dependent extrafollicular helper T cells elicit IgG production via IL-21 in systemic autoimmunity. J Exp Med 2008, 205:2873-2886

39. Deng C, Kaplan MJ, Yang J, Ray D, Zhang Z, McCune WJ, Hanash SM, Richardson BC: Decreased Ras-mitogen-activated protein kinase signaling may cause DNA hypomethylation in T lymphocytes from lupus patients. Arthritis Rheum 2001, 44:397-407.

40. Yasuda S, Stevens RL, Terada T, Takeda M, Hashimoto T, Fukae J, Horita T, Kataoka H, Atsumi T, Koike T: Defective expression of Ras guanyl nucleotide-releasing protein 1 in a subset of patients with systemic lupus erythematosus. J Immunol 2007, 179:4890-4900

41. Gorelik G, Fang JY, Wu A, Sawalha AH, Richardson B: Impaired T cell protein kinase $\mathrm{C}$ delta activation decreases ERK pathway signaling in idiopathic and hydralazine-induced lupus. J Immunol 2007, 179:5553-5563.

42. Richardson B: DNA methylation and autoimmune disease. Clin Immunol 2003, 109:72-79.

43. Krishnan S, Warke VG, Nambiar MP, Tsokos GC, Farber DL: The FcR gamma subunit and Syk kinase replace the CD3 zeta-chain and ZAP-70 kinase in the TCR signaling complex of human effector CD4 T cells. J Immunol 2003, 170:4189-4195.

44. Juang YT, Sumibcay L, Tolnay M, Wang Y, Kyttaris VC, Tsokos GC: Elf-1 binds to GGAA elements on the FcRgamma promoter and represses its expression. J Immunol 2007, 179:4884-4889.

45. Wong HK, Kammer GM, Dennis G, Tsokos GC: Abnormal NF-kappa B activity in T lymphocytes from patients with systemic lupus erythematosus is associated with decreased p65-RelA protein expression. J Immunol 1999, 163:1682-1689.

46. Karin M, Liu Z, Zandi E: AP-1 function and regulation. Curr Opin Cell Biol 1997 , 9:240-246.

47. Kyttaris VC, Juang YT, Tenbrock K, Weinstein A, Tsokos GC: Cyclic adenosine $5^{\prime}$-monophosphate response element modulator is responsible for the decreased expression of $\mathrm{c}$-fos and activator protein-1 binding in T cells from patients with systemic lupus erythematosus. J Immuno/ 2004, 173:3557-3563.

48. Mishra N, Khan IU, Tsokos GC, Kammer GM: Association of deficient type II protein kinase $A$ activity with aberrant nuclear translocation of the RII beta subunit in systemic lupus erythematosus T lymphocytes. I Immunol 2000, 165:2830-2840.

49. Katsiari CG, Kyttaris VC, Juang YT, Tsokos GC: Protein phosphatase $2 A$ is a negative regulator of IL-2 production in patients with systemic lupus erythematosus. J Clin Invest 2005, 115:3193-3204.

50. Ichinose K, Juang YT, Crispin JC, Kis-Toth K, Tsokos GC: Suppression of autoimmunity and organ pathology in lupus-prone mice upon inhibition of calcium/calmodulin-dependent protein kinase type IV. Arthritis Rheum 2011, 63:523-529.

51. Kyttaris VC, Wang Y, Juang YT, Weinstein A, Tsokos GC: Increased levels of NF-ATc2 differentially regulate CD154 and IL-2 genes in T cells from patients with systemic lupus erythematosus. I Immunol 2007, 178:1960-1966.

52. Garrett-Sinha LA, John S, Gaffen SL: IL-17 and the Th17 lineage in systemic lupus erythematosus. Curr Opin Rheumatol 2008, 20:519-525.

53. Wong CK, Lit LC, Tam LS, Li EK, Wong PT, Lam CW: Hyperproduction of IL-23 and IL-17 in patients with systemic lupus erythematosus: implications for Th17-mediated inflammation in auto-immunity. Clin Immunol 2008 , 127:385-393.
54. Crispin JC, Oukka M, Bayliss G, Cohen RA, Van Beek CA, Stillman IE, Kyttaris VC, Juang YT, Tsokos GC: Expanded double negative T cells in patients with systemic lupus erythematosus produce IL-17 and infiltrate the kidneys. J Immunol 2008, 181:8761-8766.

55. Wilson NJ, Boniface K, Chan JR, McKenzie BS, Blumenschein WM, Mattson JD, Basham B, Smith K, Chen T, Morel F, Lecron JC, Kastelein RA, Cua DJ, McClanahan TK, Bowman EP, de Waal Malefyt R: Development, cytokine profile and function of human interleukin 17-producing helper T cells. Nat Immunol 2007, 8:950-957.

56. Chen Z, Tato CM, Muul L, Laurence A, O'Shea JJ: Distinct regulation of interleukin-17 in human T helper lymphocytes. Arthritis Rheum 2007, 56:2936-2946

57. Chen Z, O'Shea JJ: Regulation of IL-17 production in human lymphocytes. Cytokine 2008, 41:71-78.

58. Harada T, Kyttaris V, Li Y, Juang YT, Wang Y, Tsokos GC: Increased expression of STAT3 in SLE T cells contributes to enhanced chemokine-mediated cell migration. Autoimmunity 2007, 40:1-8.

59. Ivanov, II, McKenzie BS, Zhou L, Tadokoro CE, Lepelley A, Lafaille JJ, Cua DJ, Littman DR: The orphan nuclear receptor RORgammat directs the differentiation program of proinflammatory IL-17+T helper cells. Cell 2006, 126:1121-1133.

60. Dong G, Ye R, Shi W, Liu S, Wang T, Yang X, Yang N, Yu X: IL-17 induces autoantibody overproduction and peripheral blood mononuclear cell overexpression of IL-6 in lupus nephritis patients. Chin Med J 2003, 116:543-548

61. Kyttaris VC, Zhang Z, Kuchroo VK, Oukka M, Tsokos GC: Cutting edge: IL-23 receptor deficiency prevents the development of lupus nephritis in C57BL/6-Ipr/Ipr mice. J Immunol 2010, 184:4605-4609.

62. Moulton VR, Tsokos GC: Alternative splicing factor/splicing factor 2 regulates the expression of the zeta subunit of the human T cell receptorassociated CD3 complex. J Bio/ Chem 2010, 285:12490-12496.

63. Tenbrock K, Juang YT, Gourley MF, Nambiar MP, Tsokos GC: Antisense cyclic adenosine $5^{\prime}$-monophosphate response element modulator up-regulates IL-2 in T cells from patients with systemic lupus erythematosus. I Immunol 2002, 169:4147-4152.

64. Richardson B: Primer: epigenetics of autoimmunity. Nat Clin Pract Rheumatol 2007, 3:521-527.

65. Sunahori K, Juang YT, Tsokos GC: Methylation status of CpG islands flanking a cAMP response element motif on the protein phosphatase $2 \mathrm{Ac}$ alpha promoter determines CREB binding and activity. J Immunol 2009, 182:1500-1508.

66. Richardson B, Scheinbart L, Strahler J, Gross L, Hanash S, Johnson M: Evidence for impaired T cell DNA methylation in systemic lupus erythematosus and rheumatoid arthritis. Arthritis Rheum 1990, 33:1665-1673

67. Li Y, Zhao M, Yin H, Gao F, Wu X, Luo Y, Zhao S, Zhang X, Su Y, Hu N, Long H, Richardson B, Lu Q: Overexpression of the growth arrest and DNA damageinduced 45alpha gene contributes to autoimmunity by promoting DNA demethylation in lupus T cells. Arthritis Rheum 2010, 62:1438-1447.

68. Juang YT, Peoples C, Kafri R, Kyttaris VC, Sunahori K, Kis-Toth K, Fitzgerald L, Ergin S, Finnell M, Tsokos G: A systemic lupus erythematosus gene expression array in disease diagnosis and classification: a preliminary report. Lupus 2010 [Epub ahead of print].

doi:10.1186/ar325

Cite this article as: Moulton VR, Tsokos GC: Abnormalities of T cell signaling in systemic lupus erythematosus. Arthritis Research \& Therapy 2011, 13:207. 